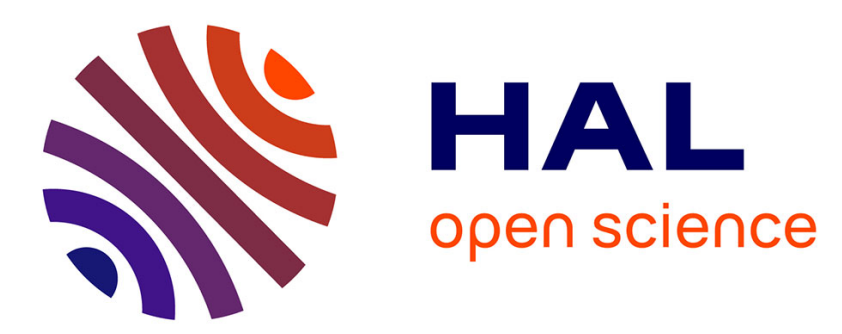

\title{
Searching for Parking in a Busy Downtown District An Agent-based Computational and Analytical Model
}

Nilankur Dutta, Alexandre Nicolas

\section{To cite this version:}

Nilankur Dutta, Alexandre Nicolas. Searching for Parking in a Busy Downtown District An Agentbased Computational and Analytical Model. 2021 International Symposium on Computer Science and Intelligent Controls (ISCSIC), Nov 2021, Rome, Italy. 10.1109/ISCSIC54682.2021.00069 . hal03368048

\section{HAL Id: hal-03368048 \\ https://hal.science/hal-03368048}

Submitted on 6 Oct 2021

HAL is a multi-disciplinary open access archive for the deposit and dissemination of scientific research documents, whether they are published or not. The documents may come from teaching and research institutions in France or abroad, or from public or private research centers.
L'archive ouverte pluridisciplinaire HAL, est destinée au dépôt et à la diffusion de documents scientifiques de niveau recherche, publiés ou non, émanant des établissements d'enseignement et de recherche français ou étrangers, des laboratoires publics ou privés. 


\title{
Searching for Parking in a Busy Downtown District An Agent-based Computational and Analytical Model

\author{
International Conference on Transport and Smart Cities 2021
}

\author{
Nilankur DUTTA and Alexandre NICOLAS \\ Institut Lumière Matière, CNRS \& Université Claude Bernard Lyon 1 \\ F-69622, Villeurbanne, France. \\ Corresponding author: Nilankur DUTTA, nilankur.dutta@univ-lyon1.fr
}

May 25, 2021

\begin{abstract}
Finding a place to park one's car is a serious issue in contemporary urban mobility. Despite the importance of the topic (30\% of cars may be cruising for parking in many large cities) and the central role given to parking policies, surprisingly little is known about the basic laws governing the search time.

We present a novel agent-based approach combining numerical simulations and theoretical considerations to model cars cruising for on-street parking in busy downtown districts. The approach is premised on the idea that, rather than parking at the first vacant spot that they encounter, drivers may be more or less prone to parking on a given spot, depending on their perceptions of its characteristics (notably its distance to their destination and its cost). This spot-specific parking probability is quantified by means of a scalar variable, the 'attractiveness'. On this premise, we show that this problem can be solved using an exact formula for the stationary state and depends on the topology of the streets. This is demonstrated by comparing our theoretical results with a stochastic in-silico model and the method is illustrated with the case of the city centre of Lyon. Finally, the relationship between the search time and the spatial modulation of the attractiveness of parking spots is explored. We find that such a modulation, which could in practice be enforced by targeted parking policies at the level of individual streets, dramatically affects the parking search time, which paves the way for a more efficient control over occupancies and cruising times in on-street parking networks.
\end{abstract}

\section{Context}

Searching for parking is often a pain in urban mobility, with an estimated average 17, 44 and 41 hours a year lost to drivers in the U.S., U.K. and Germany, respectively, in 2017. This comes at an estimated equivalent cost of $\$ 72.7$ billion, $£ 23.3$ billion and $€ 40.4$ billion in these countries in lost time, fuel, and carbon emissions [1]. The costs to the greater economy in terms of cancelled trips and psychological stress are hard to quantify, but undoubtedly substantial.

One of the manifestations of this quagmire is the number of cars cruising in search of on-street parking, which has been estimated to build up to $30 \%$ of traffic in busy downtown districts [2] in large cities. A study in Brisbane in 2015 [3] suggests that the drivers' misapprehension of the true parking costs are the major cause of this phenomenon. A recent empirical study of cruising for parking phenomenon in San Francisco [4] points out that the drivers' choice to park a longer distance away from their destination, based on their knowledge of on-street parking availability, is an important factor in regulating cruising times.

A significant portion the academic literature on parking and its relation to traffic is oriented either towards policy [5], or towards the economics of parking [6]. Much of it suggests road pricing as an effective measure to create less congested networks $[7,8]$. This work instead tackles the phenomenon of parking from a statistical physical standpoint, analysing the spatio-temporal distribution of parked cars on a network of streets. Such spatio-temporal models have been suggested by other authors $[9,10]$ in the past. In particular, the agent-based PARKAGENT model was used to model the parking dynamics of cars at night in a residential district of TelAviv [11], promoting the relevance of such models for the analysis of parking scenarios. The importance of the drivers' agency, particularly in selecting a parking strategy, has also been highlighted in other theoretical works, both on idealised one-dimensional parking lots [12], and in more physical networks or streets [13].

In this work, we present a set of analytical and computational approaches to investigate the role of the drivers' parking preferences in determining the occupancy of on-street parking spots in busy downtown districts. These 
preferences are here accounted for in terms of the 'attractiveness' of a spot, which is defined by the probability that a certain driver passing by the spot will park there, should it be vacant. This notion allows us to subsume into a single quantity the various factors governing the selection of a place to park, including its distance to the destination, cost, and intrinsic characteristics.

We implement this idea in a stochastic agent-based model and simulate it numerically to investigate the cruising phenomenon in the central district of Lyon and demonstrate the effect of modulating spot attractiveness on the on-street parking spot choices (Figure 1) and the time spent cruising for parking.

As a matter of fact, the occupancy in such a model can be solved using an exact formula and we derive an analytic formula to do so. Its accuracy is verified by comparing it with the spatial distribution of parking occupancy generated in silico (Figure 2).

\section{On-street parking in the hyper-centre of Lyon}

To illustrate our approach, we will consider the case of the central districts of Lyon (the 1st and 2nd 'arrondissements'), whose street network is available online ${ }^{1}$; the locations of the parking spots and their average occupancy street by street were kindly transmitted to us by the city council services. Generally speaking, the occupancy of parking spots results from a continuous process involving successive arrivals of car drivers with distinct destinations and parking preferences. In this short manuscript, the emphasis is put on the interplay between the network topology, the drivers' preferences, and the spatial occupation of spots, so, rather than studying the most general case, we simplify the problem and consider an influx of cars serving leisure-purpose trips in the evening with a similar destination in the vicinity of the Opera (i.e., the hyper-centric part of Lyon with the highest density of bars, restaurants, theatres, etc.), which thus acts as a 'hotspot', and similar parking preferences. Note that, upon arriving on the premises, the drivers, who are supposed to look for a spot on the street, find that many of these spots are already occupied by cars parked for a longer duration, first of which those of the residents. This initial occupation of the network is estimated using the empirical data for the occupancy in the night and a fraction of the available spots will be blocked accordingly at the beginning of the simulations.

In this network, we model the dynamics of 200 cars (which is a fairly tight upper bound for the number of leisure-motivated incoming vehicles) over a duration of 5.5 hours. These cars are injected into the network at a predefined rate through designated entry-nodes (orange bars in Figure 2A). This district is separated from the rest of the city on both the East and the West side by the two rivers Rhone and Saône, respectively, which join on the South. This geographical barrier, provides us with the physical basis of the choice on entry nodes on the eastern, southern and western side, where cars can only come in through bridges. From the northern side, we allow entrance from a high volume tunnel, the Tunnel de Croix Rousse. Route choices are implemented stochastically via prescribed turning probabilities (defined over the ensemble of cars) at each junction; in practice, we set higher probabilities for turning into a street portion whose extremity is closer to the opera hotspot (see Equation 7) in order to 'drive' the cars to their destination.

When they pass by a spot, their probability to park depends on the status (a spot must be vacant for a passing car to park in it) and attractiveness (an empty spot must be sufficiently attractive to the driver) of the spot. Since the drivers want to to park as close to the hotspot as possible, the probability to park at a vacant spot $i, A_{i}$ (三 the attractiveness of this $\operatorname{spot}^{2}$ ), is here assumed to decay exponentially with the distance to the hotspot with a characteristic length $d_{\text {walk }}$ (see Equation 6 ); $d_{\text {walk }}$ represents the preferred walking distance and will be varied in the following. Parked cars leave the network after spending a predefined time at the parked location (for details of in computational model, see Section 4.2).

\subsection{Modulating Parking Search Times via Spot Attractiveness}

In this section we investigate the phenomenon of cars in Lyon downtown districts (Figure 2).

We examine our results in the light of two factors of merit. The first one, search time, is defined as the time difference between the car first entering a disk of radius $500 \mathrm{~m}$ around the target point, and it parking. The second is walking distance, which is the distance between the parked location and the drivers destination as the crow flies. We run simulations varying the attractiveness field to three different values using the parameter $d_{\text {walk}}$, keeping everything else constant, and recording the effect on search times and walking distances (see Figure 2A).

We find that, for Lyon with $d_{\text {walk }}=100 \mathrm{~m}$, the search times in the stationary regime are around 12 minutes, with walking distances are around $450 \mathrm{~m}$

\footnotetext{
${ }^{1}$ https://data.grandlyon.com/

${ }^{2}$ The parking probabilities may actually be biased by the driver's perception of the global occupancy of the network, which affects his or her chances to find a spot. Since we consider a scenario with a fixed global occupancy, this aspect is overlooked in this short manuscript.
} 
By changing the parameter $d_{\text {walk }}$, we are able to change the area of maximum effect of our attractiveness field. When $d_{\text {walk }}$ is doubled to $200 \mathrm{~m}$, the mean search time reduces to 7.44 minutes, with the number of cars finding parking in their first couple of minutes of search almost tripled (see Figure 2B). Meanwhile, the walking distances are increased to $500 \mathrm{~m}$ (see Figure 2C). Thus, increasing the effective area of the attractiveness field reduces the competition between cars eager to park close to their destination.

We understand, therefore, that broadening the peak of the attractiveness field operates a trade-off between search times and walking distances, enabling cars to find parking faster, but compelling them to park further from their destination (see Table 1). Forcing people to park at a wider range possible of distances from their destination (e.g., by making parking more costly around hotspots) may lead to lower cruising times and less congested networks.

\begin{tabular}{||ccc||}
\hline$d_{\text {walk }}$ & walking distance $(\mathrm{m})$ & search time $(\mathrm{min})$ \\
\hline \hline 50 & 303 & 13.1 \\
\hline 100 & 441 & 11.5 \\
\hline 200 & 525 & 7.4 \\
\hline 500 & 1242 & 3.5 \\
\hline
\end{tabular}

Table 1: Effect of a spatial modulation of the parking attractiveness field on the parking search time and the actual walking distance.

\subsection{Predicting stationary occupancies analytically}

The foregoing results were obtained by means of numerical simulations. Even though we developed an efficient algorithm which allows us to simulate the parking search of tens of thousands of cars in a matter of minutes, it is inconvenient to numerically explore all possibilities (e.g., variations of injection rates, turning probabilities, parking spot attractivenesses, etc.) and the connection between the input parameters and the output of the simulations remains opaque.

To remedy these issues, deriving an analytical formula may prove an extremely valuable asset, which may provide much deeper insight into the problem. Therefore, we have endeavoured to solve the problem analytically, under the assumption of 'equilibrium', i.e., stationarity of the process, and arrived at a mean-field equation for the occupancy field. The calculations use as input parameters the rates of entry in the network and parked times, as well as the attractiveness field. The system is then fully defined according to the equations 5 and 3 , and the equilibrium occupancies can be determined (see Figure 1B). Details of the derivation are presented in the Methods section 4.1.

The comparison shown in Fig. 2 demonstrates that these theoretical considerations lead to results that coincide extremely well with the in silico predictions (the simulations were allowed to run to equilibrium, and the position and parked status of all cars were recorded at predefined intervals); also see Figure 1A. We find that the average error between the analytical prediction and the computational observation is of the order of $10^{-3}$ (see Figure 2D)

Note that, in this particular example, we have considered all allowed turns within our network to occur with a probability that hinges on the distance between the chosen street end and the driver's destination (see Equation 7). However, the equations remain valid for other route chocies, i.e., other forms of turning probabilities, including fully deterministic trajectories. 


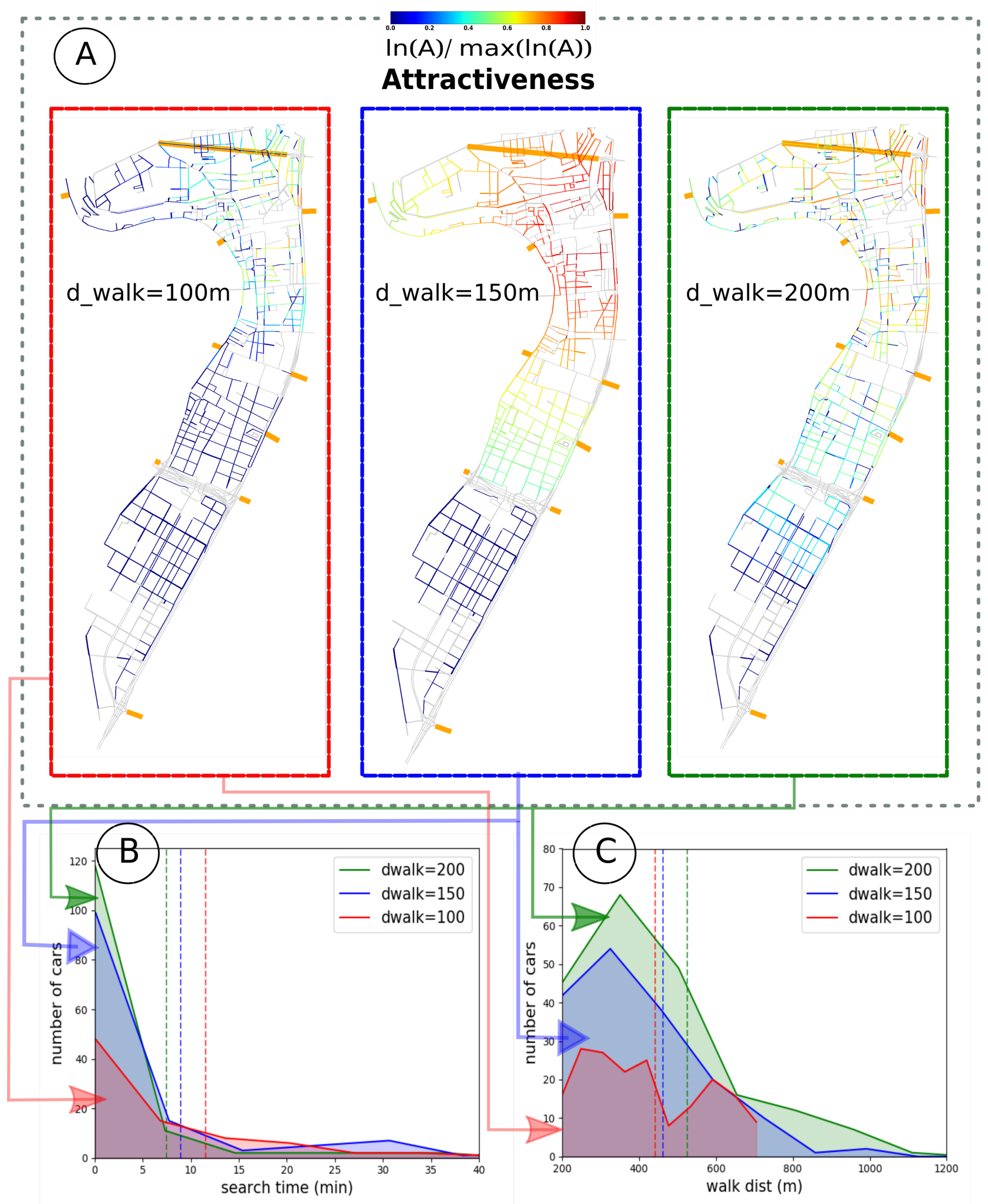

Figure 1: Modulating search times through spot attractiveness

Panel A: Three different attractiveness fields (i), (ii), (iii), obtained by modulating $d_{\text {walk }}=100 \mathrm{~m}, 150 \mathrm{~m}, 200 \mathrm{~m}$ in red, blue, and green respectively

Panel B: Distributions of search times for each sub-panel in A, with the average shown as a dashed vertical line in each case. By modulating $d_{\text {walk }}$, the mean search time is reduced from $13 \mathrm{~min}$ in (i), to $7 \mathrm{~min}$ in (iii)

Panel C: Distributions distance between parked position and destination (walkdist) each sub-panel in A, with the average shown in dashed vertical lines in each case. for less peaked attractiveness distributions, drivers park further and further from their destinations increasing from maximum distance of $762 \mathrm{~m}$ in (i) to $1718 \mathrm{~m}$ in (iii) (not shown in plot), thereby reducing local congestion. 

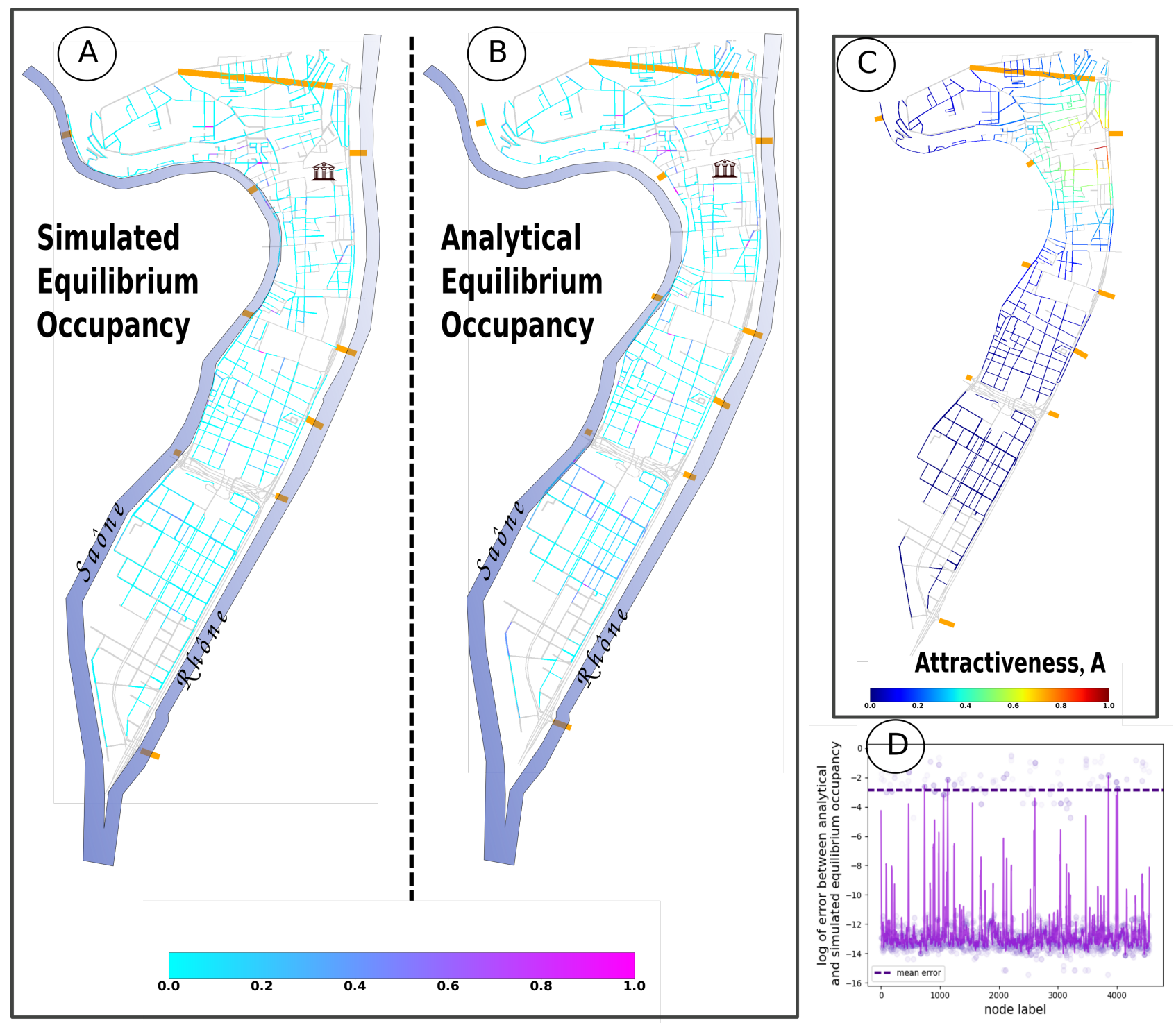

Figure 2: Predicting equilibrium street occupancy with a mathematical model:

Panels A, B: Simulated and analytical stationary parking occupancies of streets in downtown Lyon. The hotspot (the opera) is indicated by the icon, and the entry streets into the simulation are indicated by orange bars

Panel C: Spot attractiveness $A$, averaged over street portions

Panel D: Error (difference between Panels A and B, by street portions). Note the logarithmic scale. 


\section{Discussion}

Our method provides a novel mathematical framework to analyse parking in networks. We posit that describing the drivers' perception of the characteristics of a parking spot in terms of a scalar attractiveness offers a convenient way to quantitatively investigate the effect of parking choices. Not only does this provide an variable that subsumes the most important causes of the drivers agency (distance to destination, cost, etc.), but it also gives access to a mathematically rigorous derivation of the on-street occupancies with no extra knowledge except the geometry of the region of interest, the average arrival and departure rates, and the average turning probabilities, as shown here in the simplified case of cars aiming for the same goal.

In dense urban conditions, where competition for parking increases search-times and potentially causes traffic delays, it has been shown that drivers prefer on-street parking primarily due to the price premium incurred off street $[2,14]$. Parking pricing policies have been suggested by many experts as a means to alleviate the negative externalities of parking; data from studies in Beijing [7] and New Delhi [8] even suggest that parking price is one of the main determinants of the duration of parking. Thus it is important to tailor parking pricing policies to local circumstances.

We have presented spot attractiveness as a proxy variable for parking price determination, and presented how it can be used to modulate the on-street occupancies in Lyon. Thus, this work demonstrates as possible method through which empirical knowledge of parking data, and the geometry of streets, might guide city-planners towards parking pricing policies that meet their goals for on-street occupancies and cruising times.

In the real world, this could involve strategies such as differential parking pricing at the level of streets, with the ability to rapidly update prices to meet traffic demands, and the transparent communication of pricing changes to all road users.

Though in this work we have constrained ourselves to a unique attractiveness field for all users, our model is readily extensible to multiple such fields: for instance, for drivers going to different destinations, or for drivers with different psychological thresholds to parking at a certain spots. In such cases, the analytical model takes as an extra parameter the ratio of different types of cars introduced in the systems, and remains exactly solvable.

Our method is versatile enough to be used in arbitrarily different networks, and easily adapted to model off-street parking. Thus, we show that, provided certain variables like attractiveness and injection and departure rates are known, the parking occupancies can be determined in a wide array of on-street networks.

\section{Acknowledgments}

We thank Y. Pachot, C. Marolleau, and N. Keller-Mayaud from the City Council services of Lyon for giving us access to empirical data about parking, as well as L. Billaud from the City council services of Soissons. This work has been funded by an Impulsion grant from IDEXLYON (2020-2021).

\section{Methods}

\subsection{Analytical Model for Parking in One Dimensional Street}

This section is dedicated to mathematical details about the formulas giving the stationary parking occupancy.

Consider a one dimensional parking lot with $L$ spots, with distance $l$ between them, labelled by an integer index $x \mid x \in\{0, L-1\}$. Defining here the relevant variables : the field of attractiveness as $A(x)$, the probability of a spot being occupied as $n(x)$, and its inverse $\tilde{n}(x)=1-n(x)$,

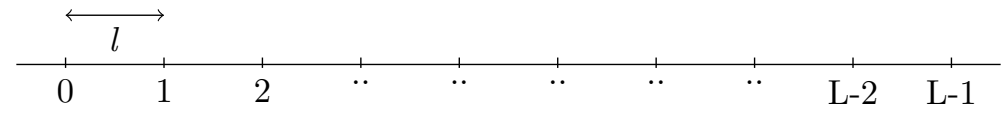

Figure 3: Schematic of 1D parking lot

Then the probability of parking at any spot $n(x)$ can be written as:

$$
P(x)=\tilde{n}^{\prime}(x) \prod_{x^{\prime}=0}^{x-1}\left(\tilde{n}\left(x^{\prime}\right)+\tilde{n^{\prime}}\left(x^{\prime}\right)\right)
$$

where the first term on the right-hand side, $\tilde{n}^{\prime}(x) \equiv \tilde{n}(x) A(x)$, is the probability of parking at a spot $x$ multiplied by the probability that it is vacant, and the second $\tilde{n}\left(x^{\prime}\right)$ and third terms $\tilde{n}^{\prime}\left(x^{\prime}\right) \equiv p\left(x^{\prime}\right)\left(1-A\left(x^{\prime}\right)\right)$ 
are the probabilities of all previous parking spots being either full or not attractive enough for the driver, respectively.

Equation 1 can be simplified as :

$$
P(x)=\tilde{n}^{\prime}(x) \prod_{x^{\prime}=0}^{x-1}\left(1-\tilde{n}\left(x^{\prime}\right) A\left(x^{\prime}\right)\right)
$$

In the continuous limit, the change in occupancy of each spot is defined by the differential equation:

$$
\frac{d n(x)}{d t}=I P(x)-D n(x)
$$

where $\mathrm{I}$ is the rate of entry of cars into the street, and D is the rate at which parked cars depart from their spots. So in the stationary state:

$$
n(x)=\frac{I}{D} P(x)
$$

The generalisation to networks is straightforward, by defining the transition probability from spot $x$ to spot $x^{\prime}$ as $T_{x}^{x^{\prime}}$. Then the equation 2 takes on this as an extra term, and a sum over all paths $\pi$ from $i$ :

$$
P(x \mid i)=\sum_{\pi}\left[I_{i} \prod_{x^{\prime} \in X}\left(1-\tilde{n}\left(x^{\prime}\right) A\left(x^{\prime}\right)\right) T_{x}^{x^{\prime}}\right] \tilde{n}^{\prime}(x)
$$

where $\mathrm{i}$ is the set of all 'entry nodes' where cars enter the network with probability $I_{i}$

\subsection{Details of the in-silico model}

To demonstrate our method, we simulate evening on-street parking in the downtown commercial districts of (our hometown) Lyon: the 1st and 2nd arrondissements. The network consists of 1835 directional street portions between junctions (a two-way street portion would consist of two such directional portions), as well as 8778 parking spots distributed over these street portions. Details of the parking spots are provided to us by the city office of Lyon.

The departure rate, $D$ is chosen an exponential distribution peaked around the mean parking duration of 2 hours, based on known parking durations in these districts. The cars are kept in the simulation system until they park, after which they are removed based on $D$.

Since we want to model the dynamics cars intent on parking near the opera, we pick the attractiveness field $A_{s}(\mathbf{x})$ for a street beginning at the coordinate $\mathbf{x}$ an exponential centered on the opera, (see Figure 1), given by the equation:

$$
A_{s}(\mathbf{x})=\exp -\frac{\left|\mathbf{x}-\mathbf{x}_{\mathbf{o}}\right|}{d_{\text {walk }}}
$$

where $\mathbf{x}_{\mathbf{o}}$ is the location of the opera, and $d_{\text {walk }}$ is a parameter that gives the distance from the opera at which, the attractiveness falls to 0.367 ( or $e^{-1}$ ) times the maximal attractiveness.

To have more 'intelligent' drivers that are driven towards their destination, we also implement, (for the analysis in Section 2.1) turning probabilities at junctions. For instance, at a junction with $n$ possible choices street choices, $\mathbf{S}=\left\{s_{1}, s_{2}, \ldots, s_{n}\right\}$ the transition from street $s^{\prime} \longrightarrow s_{k}$ is given as:

$$
T_{s_{k}}^{s^{\prime}}(\mathbf{x})=\frac{\exp -\left|\mathbf{x}_{\mathbf{s}_{\mathbf{k}}}-\mathbf{x}_{\mathbf{o}}\right|}{\sum_{s_{i} \in \mathbf{S}} \exp -\left|\mathbf{x}_{\mathbf{s}_{\mathbf{i}}}-\mathbf{x}_{\mathbf{o}}\right|}
$$

where $\mathbf{x}_{\mathbf{S}_{\mathbf{i}}}$ is the location of the end node of street $s_{i}$, and all other variables retain their usual significance. Cars can exit from the network only if they arrive at a cul-de-sac street, where a U-turn is not allowed.

The only other required parameters are the rate of the arrival of new cars aiming to park at the hotspot into the simulation volume $I_{i}$. This is chosen to be 72 cars per hour.

In all examples used in this work, the simulation is performed with a time step of 1 second and runs for $2 \cdot 10^{5}$ seconds (or 5 hours and 34 minutes). The first $10^{5}$ seconds, including a transient regime, are discarded and the data are only collected in the second half of this period, at 100 second intervals. Thus, in all simulations presented in this work, about 200 cars are injected over the total simulation time. Simulating this time interval only takes 2.32 seconds of real computational time with our highly economical algorithm. 


\section{References}

[1] G. Cookson and B. Pishue, "The impact of parking pain in the us, uk and germany," Hg. v. INRIX Research. Online verfügbar unter http://inrix. com/research/parking-pain/, zuletzt geprüft am, vol. 21, p. 2018, 2017.

[2] D. C. Shoup, "Cruising for parking," Transport policy, vol. 13, no. 6, pp. 479-486, 2006.

[3] J. Lee, D. Agdas, and D. Baker, "Cruising for parking: New empirical evidence and influential factors on cruising time," Journal of Transport and Land Use, vol. 10, no. 1, pp. 931-943, 2017.

[4] A. Millard-Ball, R. C. Hampshire, and R. Weinberger, "Parking behaviour: The curious lack of cruising for parking in san francisco," Land Use Policy, vol. 91, p. 103918, 2020.

[5] D. C. Shoup, The high cost of free parking. Routledge, 2021.

[6] E. Calthrop, S. Proost, and K. Van Dender, "Parking policies and road pricing," Urban studies, vol. 37, no. 1 , pp. $63-76,2000$.

[7] F. Zong and M. Wang, "Understanding parking decisions with a bayesian network," Transportation Planning and Technology, vol. 38, no. 6, pp. 585-600, 2015.

[8] J. Parmar, P. Das, and S. M. Dave, "Study on demand and characteristics of parking system in urban areas: A review," Journal of Traffic and Transportation Engineering (English Edition), vol. 7, no. 1, pp. 111-124, 2020 .

[9] N. Geroliminis, "Cruising-for-parking in congested cities with an mfd representation," Economics of Transportation, vol. 4, no. 3, pp. 156-165, 2015.

[10] R. Arnott and J. Rowse, "Modeling parking," Journal of urban economics, vol. 45, no. 1, pp. 97-124, 1999.

[11] N. Levy, K. Martens, and I. Benenson, "Exploring cruising using agent-based and analytical models of parking," Transportmetrica A: Transport Science, vol. 9, no. 9, pp. 773-797, 2013.

[12] P. Krapivsky and S. Redner, "Simple parking strategies," Journal of Statistical Mechanics: Theory and Experiment, vol. 2019, no. 9, p. 093404, 2019.

[13] I. Benenson, K. Martens, and S. Birfir, "Parkagent: An agent-based model of parking in the city," Computers, Environment and Urban Systems, vol. 32, no. 6, pp. 431-439, 2008.

[14] E. Inci and R. Lindsey, "Garage and curbside parking competition with search congestion," Regional Science and Urban Economics, vol. 54, pp. 49-59, 2015. 